Acta Crystallographica Section E

Structure Reports

Online

ISSN 1600-5368

John A. Cowan, ${ }^{\text {** Judith A. K. }}$ Howard, ${ }^{\text {Horst Puschmann }}{ }^{\mathrm{a}}$ and Ian D. Williams ${ }^{b}$

${ }^{a}$ Department of Chemistry, University of Durham, Durham DH1 3LE, England, and ${ }^{\mathbf{b}}$ Department of Chemistry, Hong Kong University of Science and Technology, Clear Water Bay, Hong Kong, People's Republic of China

Correspondence e-mail: j.a.cowan@dl.ac.uk

Key indicators

Single-crystal X-ray study

$T=100 \mathrm{~K}$

Mean $\sigma(\mathrm{C}-\mathrm{C})=0.004 \AA$

$R$ factor $=0.070$

$w R$ factor $=0.193$

Data-to-parameter ratio $=13.9$

For details of how these key indicators were automatically derived from the article, see http://journals.iucr.org/e.

\title{
Short $\mathrm{N} \cdots \mathrm{O}$ hydrogen bonds in the 1:1 adduct of 4,4'-bipyridyl and oxalic acid
}

Oxalic acid, $\mathrm{C}_{2} \mathrm{O}_{4} \mathrm{H}_{2}$, and 4,4'-bipyridyl, $\mathrm{C}_{10} \mathrm{H}_{8} \mathrm{~N}_{2}$, crystallize in a 1:1 ratio. The asymmetric unit consists of one oxalic acid (OXA) molecule and one 4,4'bipyridyl (BPY) molecule in general positions, together with one half-OXA molecule and one half-BPY molecule; the latter two molecules are centrosymmetric. The molecules are linked in two parallel independent chains by strong $\mathrm{O}-\mathrm{H} \cdots \mathrm{N}$ hydrogen bonds. In one chain there is one independent $\mathrm{O}-\mathrm{H} \cdots \mathrm{N}$ hydrogen bond $[\mathrm{N} \cdots \mathrm{O}=2.557(3) \AA]$ and the molecules lie on centres of symmetry and are therefore constrained to have planar central portions. The second chain contains two independent $\mathrm{O}-$ $\mathrm{H} \cdots \mathrm{N}$ hydrogen bonds $[\mathrm{O} \cdots \mathrm{N}=2.549$ (3) and 2.581 (3) $\AA$ ] and both molecules are twisted about their central bonds.

\section{Comment}

Temperature-dependent proton migration has recently been observed in short N...O hydrogen bonds between carboxylic acid and pyridyl groups (Cowan et al., 2003, 2005). It occurred to us that 4,4'-bipyridyl (BPY) and oxalic acid (OXA) would be likely to co-crystallize in the same intermolecular configuration, but hopefully with only one independent $\mathrm{N}-\mathrm{H} \cdots \mathrm{O}$ hydrogen bond in the asymmetric unit. We present here the crystal structure of the title 1:1 adduct of BPY and OXA, (I).

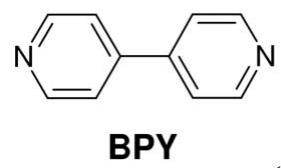

(I)

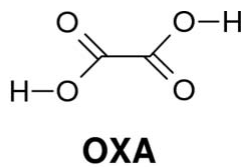

The asymmetric unit of (I) consists of one oxalic acid (OXA) molecule and one 4,4'-bipyridyl (BPY) molecule in general positions, together with one half-OXA molecule and one half-BPY molecule; the latter two molecules are centrosymmetric. BPY and OXA crystallize to form two similar independent one-dimensional chains. In both chains the OXA and BPY molecules are linked together by strong $\mathrm{O}-\mathrm{H} \cdots \mathrm{N}$ hydrogen bonds in concert with $\mathrm{C}-\mathrm{H} \cdots \mathrm{O}$ hydrogen bonds (Table 1). A similar configuration is often observed in cocrystals of BPY and carboxylic acids, for example in the cocrystals of BPY with fumaric acid (Chatterjee et al., 1998), phosphonoacetic acid (Bowes et al., 2003) and malonic acid (Pedireddi et al., 1998). That one-dimensional tapes are formed is unsurprising considering the co-crystals of BPY with 2,5-dihydroxybenzoquinone (Cowan et al., 2001), squaric acid (Reetz et al., 1994) and 2,5-dichloro-3,6-dihydroxybenzoquinone (Zaman et al., 1999), which all have a similar
Received 16 January 2007 Accepted 31 January 2007
(C) 2007 International Union of Crystallography All rights reserved 

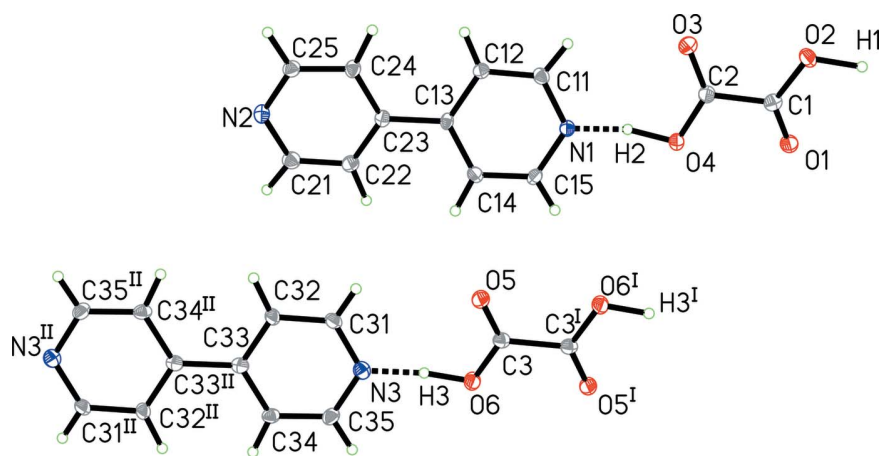

Figure 1

The structures of segments of both independent chain, with the atomnumbering scheme. Displacement ellipsoids are drawn at the $50 \%$ probability level. Dashed lines indicate hydrogen bonds and $\mathrm{H}$ atoms are shown as small spheres of arbitrary radii. [Symmetry codes: (I) $2-x$, $2-y, 1-z$; (II) $3-x, 1-y, 1-z$.]

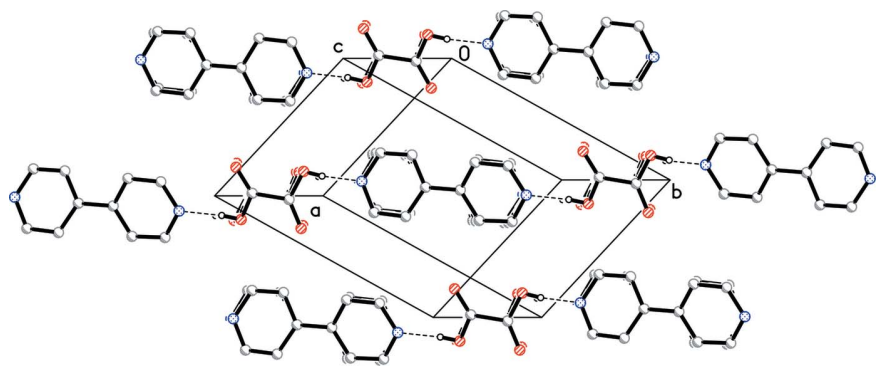

Figure 2

A packing diagram for (I), illustrating the molecular chains. All $\mathrm{H}$ atoms, except those involved in strong hydrogen bonds, have been omitted for clarity.

arrangement of $\mathrm{O}$ atoms to OXA and which all form onedimensional tapes.

The two types of chains are distinguished by the twists within the molecules. In one chain, the planes of the pyridyl rings of the BPY molecule are twisted by $23.75(6)^{\circ}$ with respect to each other and there is a twist of $5.35(11)^{\circ}$ between the carboxylic acid groups of the OXA molecule, while in the other chain, the OXA and BPY molecules lie on centres of symmetry and are consequently both have planar central portions. Both chains lie in the $a b$ plane and propagate in the [11̄0] direction (Fig. 2). The chains are linked by $\mathrm{C}-\mathrm{H} \cdots \mathrm{O}$ hydrogen bonds into parallel planes. One set of planes consists of only flat molecules and the other consists of only twisted molecules. The only significant interaction linking the planes is a $\mathrm{C}-\mathrm{H} \cdots \mathrm{O}$ hydrogen bond $(\mathrm{C} 11 \cdots \mathrm{O} 3)$ between adjacent planes of twisted molecules.

There are three similar $\mathrm{O}-\mathrm{H} \cdots \mathrm{N}$ hydrogen bonds in the structure of (I). Although the $\mathrm{H}$-atom positions were constrained in the final refinement, $\mathrm{O}-\mathrm{H}$ distances between 1.15 and $1.25 \AA$ in earlier free refinements hint that the true $\mathrm{H}$ atom positions may be close to the centres of the hydrogen bonds. The graph produced by Steiner (2002) from neutron diffraction data of $\mathrm{N} \cdots \mathrm{O}$ hydrogen bonds suggests that the $\mathrm{O}-\mathrm{H}$ distance becomes significantly elongated when the $\mathrm{N}-$ O distance is below $\sim 2.6 \AA$. Although not as short as the hydrogen bonds in which temperature-dependent proton migration has been observed, the $\mathrm{N} \cdots \mathrm{O}$ distances in the three hydrogen bonds in (I) are all below $\sim 2.6 \AA$. Therefore, a large elongation of the $\mathrm{O}-\mathrm{H}$ bond is expected and the $\mathrm{H}$-atom position may be temperature-dependent. Neutron diffraction is required for accurate $\mathrm{H}$-atom positions.

\section{Experimental}

Equimolar quantities of BPY and OXA were dissolved in methanol. Crystals of (I) suitable for X-ray structure determination were prepared by slow evaporation of the solvent at room temperature.

Crystal data
$\mathrm{C}_{10} \mathrm{H}_{8} \mathrm{~N}_{2} \cdot \mathrm{C}_{2} \mathrm{H}_{2} \mathrm{O}_{4}$

$M_{r}=246.22$

Triclinic, $P \overline{1}$

$a=8.7365(18) \AA$

$b=9.9154(19) \AA$

$c=10.380(2) \AA$

$\alpha=100.253(10)^{\circ}$

$\beta=105.349(11)^{\circ}$

$\gamma=107.569(10)^{\circ}$

\section{Data collection}

Bruker SMART CCD area-detector diffractometer

$\omega$ scans

Absorption correction: none

8434 measured reflections

\section{Refinement}

Refinement on $F^{2}$

$R\left[F^{2}>2 \sigma\left(F^{2}\right)\right]=0.070$

$w R\left(F^{2}\right)=0.193$

$S=1.09$

3601 reflections

259 parameters

Only H-atom displacement para-

meters refined

$$
\begin{aligned}
& V=793.5(3) \AA^{3} \\
& Z=3 \\
& D_{x}=1.546 \mathrm{Mg} \mathrm{m}^{-3} \\
& \text { Mo } K \alpha \text { radiation }^{-1} \\
& \mu=0.12 \mathrm{~mm}^{-1} \\
& T=100(2) \mathrm{K} \\
& \text { Block, brown } \\
& 0.3 \times 0.2 \times 0.15 \mathrm{~mm}
\end{aligned}
$$

3601 independent reflections 2523 reflections with $I>2 \sigma(I)$ $R_{\text {int }}=0.058$

$\theta_{\max }=27.5^{\circ}$
Table 1

Hydrogen-bond geometry $\left(\AA,{ }^{\circ}\right)$.

\begin{tabular}{lllll}
\hline$D-\mathrm{H} \cdots A$ & $D-\mathrm{H}$ & $\mathrm{H} \cdots A$ & $D \cdots A$ & $D-\mathrm{H} \cdots A$ \\
\hline $\mathrm{O} 2-\mathrm{H} 1 \cdots \mathrm{N} 2^{\mathrm{i}}$ & 0.82 & 1.77 & $2.586(3)$ & 174 \\
$\mathrm{O} 4-\mathrm{H} 2 \cdots \mathrm{N} 1$ & 0.82 & 1.74 & $2.553(3)$ & 174 \\
$\mathrm{O} 6-\mathrm{H} 3 \cdots \mathrm{N} 3$ & 0.82 & 1.74 & $2.560(3)$ & 174 \\
$\mathrm{C} 11-\mathrm{H} 11 \cdots \mathrm{O} 3^{\text {ii }}$ & 0.93 & 2.59 & $3.270(4)$ & 130 \\
$\mathrm{C}^{\text {iii }} 2-\mathrm{H} 22 \cdots 3^{\text {ii }}$ & 0.93 & 2.45 & $3.375(3)$ & 172 \\
$\mathrm{C}^{2} 4-\mathrm{H} 24 \cdots 1^{\text {iv }}$ & 0.93 & 2.40 & $3.326(4)$ & 171 \\
$\mathrm{C} 25-\mathrm{H} 25 \cdots 4^{\text {iv }}$ & 0.93 & 2.48 & $3.216(3)$ & 136 \\
$\mathrm{C} 32-\mathrm{H} 32 \cdots 5^{\mathrm{v}}$ & 0.93 & 2.56 & $3.418(3)$ & 153 \\
$\mathrm{C} 34-\mathrm{H} 34 \cdots 5^{\text {iii }}$ & 0.93 & 2.52 & $3.373(3)$ & 153 \\
$\mathrm{C} 35-\mathrm{H} 35 \cdots \mathrm{O}^{\text {vi }}$ & 0.93 & 2.57 & $3.207(3)$ & 126 \\
\hline
\end{tabular}

Symmetry codes: (i) $x-1, y+1, z$; (ii) $-x,-y+1,-z$; (iii) $x+1, y, z$; (iv) $x, y-1, z$; (v) $-x+2,-y+1,-z+1$; (vi) $-x+3,-y+2,-z+1$.

All $\mathrm{H}$ atoms were located in a difference Fourier map and then repositioned in idealized locations, with $\mathrm{O}-\mathrm{H}=0.82 \AA$ and $\mathrm{C}-\mathrm{H}=$ $0.93 \AA$. They were refined with their coordinates, but not their isotropic displacement parameters, riding on their parent atoms.

Data collection: SMART (Bruker, 1998); cell refinement: SMART; data reduction: SAINT (Bruker, 1998); program(s) used to solve structure: SHELXS97 (Sheldrick, 1997); program(s) used to refine 


\section{organic papers}

structure: SHELXL97 (Sheldrick, 1997); molecular graphics: SHELXTL/PC (Sheldrick, 1999); software used to prepare material for publication: SHELXL97.

\section{References}

Bowes, K. F., Ferguson, G., Lough, A. J., Zakaria, C. M. \& Glidewell, C. (2003). Acta Cryst. B59, 87-99.

Bruker (1998). SMART and SAINT. Bruker AXS Inc., Madison, Wisconsin, USA.

Chatterjee, S., Pedireddi, V. R. \& Rao, C. N. R. (1998). Tetrahedron Lett. 39, 2843-2846.

Cowan, J. A., Howard, J. A. K. \& Leech, M. A. (2001). Acta Cryst. C57, 302303.
Cowan, J. A., Howard, J. A. K., McIntyre, G. J., Lo, S. M.-F. \& Williams, I. D. (2003). Acta Cryst. B59, 794-801.

Cowan, J. A., Howard, J. A. K., McIntyre, G. J., Lo, S. M.-F. \& Williams, I. D. (2005). Acta Cryst. B61, 724-730.

Pedireddi, V. R., Chatterjee, S., Ranganathan, A. \& Rao, C. N. R. (1998). Tetrahedron, 54, 9457-9474.

Reetz, M. T., Höger, S. \& Harms, K. (1994). Angew. Chem. Int. Ed. Engl. 33 181-183.

Sheldrick, G. M. (1997). SHELXS97 and SHELXL97. University of Göttingen, Germany.

Sheldrick, G. M. (1999). SHELXTL/PC. Version 5.10 for Windows NT. Bruker AXS Inc., Madison, Wisconsin, USA.

Steiner, T. (2002). Angew. Chem. Int. Ed. 41, 48-76.

Zaman, M. B., Tomura, M. \& Yamashita, Y. (1999). Chem. Commun. pp. 9991000 


\section{supporting information}

Acta Cryst. (2007). E63, o1240-o1242 [https://doi.org/10.1107/S1600536807005156]

\section{Short $\mathrm{N} \cdots \mathrm{O}$ hydrogen bonds in the 1:1 adduct of 4,4'-bipyridyl and oxalic acid}

John A. Cowan, Judith A. K. Howard, Horst Puschmann and lan D. Williams

4,4'-bipyridyl-oxalic acid (1/1)

Crystal data

$\mathrm{C}_{10} \mathrm{H}_{8} \mathrm{~N}_{2} \cdot \mathrm{C}_{2} \mathrm{H}_{2} \mathrm{O}_{4}$

$Z=3$

$M_{r}=246.22$

Triclinic, $P \overline{1}$

Hall symbol: -P 1

$a=8.7365$ (18) $\AA$

$b=9.9154(19) \AA$

$c=10.380(2) \AA$

$\alpha=100.253(10)^{\circ}$

$\beta=105.349(11)^{\circ}$

$F(000)=384$

$D_{\mathrm{x}}=1.546 \mathrm{Mg} \mathrm{m}^{-3}$

Mo $K \alpha$ radiation, $\lambda=0.71073 \AA$

Cell parameters from 874 reflections

$\theta=10.1-19.0^{\circ}$

$\mu=0.12 \mathrm{~mm}^{-1}$

$T=100 \mathrm{~K}$

$\gamma=107.569(10)^{\circ}$

Block, brown

$V=793.5(3) \AA^{3}$

$0.3 \times 0.2 \times 0.15 \mathrm{~mm}$

\section{Data collection}

Bruker SMART CCD area-detector diffractometer

Radiation source: fine-focus sealed tube Graphite monochromator

$\omega$ scans

8434 measured reflections

3601 independent reflections

2523 reflections with $I>2 \sigma(I)$

$R_{\text {int }}=0.058$

$\theta_{\max }=27.5^{\circ}, \theta_{\min }=2.1^{\circ}$

$h=-11 \rightarrow 9$

$k=-12 \rightarrow 12$

$l=-13 \rightarrow 13$

\section{Refinement}

Refinement on $F^{2}$

Least-squares matrix: full

$R\left[F^{2}>2 \sigma\left(F^{2}\right)\right]=0.070$

$w R\left(F^{2}\right)=0.193$

$S=1.09$

3601 reflections

259 parameters

0 restraints

Primary atom site location: structure-invariant direct methods

Secondary atom site location: difference Fourier map

Hydrogen site location: inferred from neighbouring sites

Only $\mathrm{H}$-atom displacement parameters refined

$w=1 /\left[\sigma^{2}\left(F_{\mathrm{o}}^{2}\right)+(0.0821 P)^{2}+0.9233 P\right]$ where $P=\left(F_{\mathrm{o}}{ }^{2}+2 F_{\mathrm{c}}{ }^{2}\right) / 3$

$(\Delta / \sigma)_{\max }<0.001$

$\Delta \rho_{\max }=0.53$ e $\AA^{-3}$

$\Delta \rho_{\min }=-0.34$ e $\AA^{-3}$

Special details

Geometry. All e.s.d.'s (except the e.s.d. in the dihedral angle between two 1.s. planes) are estimated using the full covariance matrix. The cell e.s.d.'s are taken into account individually in the estimation of e.s.d.'s in distances, angles and torsion angles; correlations between e.s.d.'s in cell parameters are only used when they are defined by crystal symmetry. An approximate (isotropic) treatment of cell e.s.d.'s is used for estimating e.s.d.'s involving 1.s. planes. 
Refinement. Refinement of $F^{2}$ against ALL reflections. The weighted $R$-factor $w R$ and goodness of fit $S$ are based on $F^{2}$, conventional $R$-factors $R$ are based on $F$, with $F$ set to zero for negative $F^{2}$. The threshold expression of $F^{2}>\sigma\left(F^{2}\right)$ is used only for calculating $R$-factors(gt) etc. and is not relevant to the choice of reflections for refinement. $R$-factors based on $F^{2}$ are statistically about twice as large as those based on $F$, and $R$ - factors based on ALL data will be even larger.

Fractional atomic coordinates and isotropic or equivalent isotropic displacement parameters $\left(\hat{A}^{2}\right)$

\begin{tabular}{|c|c|c|c|c|}
\hline & $x$ & $y$ & $z$ & $U_{\text {iso }} * / U_{\text {eq }}$ \\
\hline $\mathrm{C} 1$ & $0.0631(4)$ & $0.9499(3)$ & 0.1702 (3) & $0.0157(6)$ \\
\hline $\mathrm{O} 1$ & $0.1464(3)$ & $1.0696(2)$ & $0.1636(2)$ & $0.0204(5)$ \\
\hline $\mathrm{O} 2$ & $-0.0932(3)$ & $0.9108(2)$ & $0.1751(2)$ & $0.0195(5)$ \\
\hline H1 & -0.1252 & 0.9805 & 0.1745 & $0.080(18)^{*}$ \\
\hline $\mathrm{C} 2$ & $0.1318(4)$ & $0.8219(3)$ & $0.1705(3)$ & $0.0150(5)$ \\
\hline $\mathrm{O} 3$ & 0.0336 & $0.6941(2)$ & $0.1412(2)$ & $0.0247(5)$ \\
\hline $\mathrm{O} 4$ & $0.2955(2)$ & $0.8668(2)$ & 0.2015 (2) & $0.0192(5)$ \\
\hline $\mathrm{H} 2$ & 0.3248 & 0.7954 & 0.1933 & $0.10(2)^{*}$ \\
\hline N1 & 0.4089 & $0.6576(2)$ & $0.1828(2)$ & $0.0128(5)$ \\
\hline C11 & $0.3087(4)$ & $0.5142(3)$ & $0.1246(3)$ & $0.0148(6)$ \\
\hline H11 & 0.1909 & 0.4881 & 0.0909 & $0.021(8)^{*}$ \\
\hline $\mathrm{C} 12$ & $0.3763(3)$ & 0.4040 & $0.1134(3)$ & $0.0136(5)$ \\
\hline H12 & 0.3046 & 0.3057 & 0.0731 & $0.020(8)^{*}$ \\
\hline $\mathrm{C} 13$ & $0.5547(3)$ & 0.4431 & 0.1638 & $0.0112(5)$ \\
\hline $\mathrm{C} 14$ & 0.6565 & $0.5934(3)$ & $0.2216(3)$ & $0.0144(5)$ \\
\hline H14 & 0.7748 & 0.6235 & 0.2536 & $0.022(8)^{*}$ \\
\hline $\mathrm{C} 15$ & 0.5800 & $0.6968(3)$ & 0.2308 & $0.0145(5)$ \\
\hline H15 & 0.6485 & 0.7959 & 0.2712 & $0.014(7)^{*}$ \\
\hline N2 & 0.7855 & $0.1180(2)$ & $0.1646(2)$ & $0.0156(5)$ \\
\hline $\mathrm{C} 21$ & $0.8754(4)$ & 0.2565 & 0.1681 (3) & $0.0178(6)$ \\
\hline $\mathrm{H} 21$ & 0.9884 & 0.2805 & 0.1721 & $0.024(9)^{*}$ \\
\hline $\mathrm{C} 22$ & $0.8046(4)$ & $0.3642(3)$ & 0.1659 (3) & $0.0163(6)$ \\
\hline $\mathrm{H} 22$ & 0.8694 & 0.4587 & 0.1680 & $0.034(10)^{*}$ \\
\hline $\mathrm{C} 23$ & $0.6351(3)$ & 0.3295 & $0.1604(3)$ & $0.0130(5)$ \\
\hline $\mathrm{C} 24$ & $0.5416(4)$ & $0.1853(3)$ & $0.1546(3)$ & $0.0144(5)$ \\
\hline $\mathrm{H} 24$ & 0.4277 & 0.1574 & 0.1485 & $0.023(9)^{*}$ \\
\hline $\mathrm{C} 25$ & $0.6229(4)$ & $0.0842(3)$ & $0.1581(3)$ & $0.0155(6)$ \\
\hline $\mathrm{H} 25$ & 0.5613 & -0.0113 & 0.1559 & $0.012(7)^{*}$ \\
\hline $\mathrm{C} 3$ & $1.0356(3)$ & $0.9366(3)$ & 0.5023 & $0.0142(5)$ \\
\hline $\mathrm{O} 5$ & $0.9471(2)$ & $0.8154(2)$ & $0.5031(2)$ & $0.0185(4)$ \\
\hline O6 & $1.1925(2)$ & $0.9765(2)$ & $0.5046(2)$ & $0.0192(5)$ \\
\hline $\mathrm{H} 3$ & 1.2232 & 0.9060 & 0.5033 & $0.10(2)^{*}$ \\
\hline N3 & $1.3099(3)$ & $0.7691(2)$ & $0.5027(2)$ & $0.0155(5)$ \\
\hline $\mathrm{C} 31$ & $1.2128(4)$ & 0.6253 & 0.4680 & $0.0160(6)$ \\
\hline H31 & 1.0951 & 0.5979 & 0.4446 & $0.013(7)^{*}$ \\
\hline $\mathrm{C} 32$ & $1.2821(3)$ & $0.5163(3)$ & $0.4658(3)$ & $0.0154(6)$ \\
\hline H32 & 1.2116 & 0.4178 & 0.4415 & $0.030(9)^{*}$ \\
\hline $\mathrm{C} 33$ & $1.4596(3)$ & $0.5564(3)$ & 0.5007 (3) & $0.0132(5)$ \\
\hline C34 & $1.5585(3)$ & $0.7073(3)$ & $0.5373(3)$ & $0.0143(5)$ \\
\hline H34 & 1.6765 & 0.7387 & 0.5607 & $0.020(8)^{*}$ \\
\hline
\end{tabular}




\begin{tabular}{lllll}
$\mathrm{C} 35$ & $1.4788(4)$ & $0.8091(3)$ & $0.5382(3)$ & $0.0161(6)$ \\
$\mathrm{H} 35$ & 1.5457 & 0.9089 & 0.5645 & $0.024(9)^{*}$ \\
\hline
\end{tabular}

Atomic displacement parameters $\left(\AA^{2}\right)$

\begin{tabular}{lllllll}
\hline & $U^{11}$ & $U^{22}$ & $U^{33}$ & $U^{12}$ & $U^{13}$ & $U^{23}$ \\
\hline C1 & $0.0173(14)$ & $0.0156(13)$ & $0.0141(12)$ & $0.0053(11)$ & $0.0061(11)$ & $0.0040(9)$ \\
O1 & $0.0169(11)$ & $0.0149(9)$ & $0.0321(11)$ & $0.0053(8)$ & $0.0114(9)$ & $0.0095(8)$ \\
O2 & $0.0149(10)$ & $0.0191(10)$ & $0.0285(11)$ & $0.0085(8)$ & $0.0098(9)$ & $0.0088(8)$ \\
C2 & $0.0159(14)$ & $0.0146(13)$ & $0.0163(12)$ & $0.0063(11)$ & $0.0069(11)$ & $0.0051(10)$ \\
O3 & $0.0185(11)$ & $0.0137(10)$ & $0.0411(13)$ & $0.0053(8)$ & $0.0090(9)$ & $0.0081(8)$ \\
O4 & $0.0143(10)$ & $0.0122(9)$ & $0.0330(11)$ & $0.0064(8)$ & $0.0096(8)$ & $0.0056(8)$ \\
N1 & $0.0131(12)$ & $0.0116(10)$ & $0.0169(11)$ & $0.0049(9)$ & $0.0083(9)$ & $0.0064(8)$ \\
C11 & $0.0129(14)$ & $0.0167(13)$ & $0.0164(12)$ & $0.0061(10)$ & $0.0048(11)$ & $0.0075(10)$ \\
C12 & $0.0135(13)$ & $0.0116(12)$ & $0.0163(12)$ & $0.0041(10)$ & $0.0060(10)$ & $0.0047(9)$ \\
C13 & $0.0141(13)$ & $0.0111(12)$ & $0.0131(11)$ & $0.0069(10)$ & $0.0081(10)$ & $0.0054(9)$ \\
C14 & $0.0117(13)$ & $0.0150(12)$ & $0.0167(13)$ & $0.0056(10)$ & $0.0043(10)$ & $0.0049(10)$ \\
C15 & $0.0150(14)$ & $0.0102(12)$ & $0.0180(13)$ & $0.0043(10)$ & $0.0064(11)$ & $0.0027(9)$ \\
N2 & $0.0175(12)$ & $0.0154(11)$ & $0.0170(11)$ & $0.0081(9)$ & $0.0074(9)$ & $0.0063(8)$ \\
C21 & $0.0161(14)$ & $0.0178(13)$ & $0.0191(13)$ & $0.0046(11)$ & $0.0062(11)$ & $0.0066(10)$ \\
C22 & $0.0158(14)$ & $0.0137(12)$ & $0.0199(13)$ & $0.0046(11)$ & $0.0068(11)$ & $0.0065(10)$ \\
C23 & $0.0156(13)$ & $0.0140(12)$ & $0.0099(11)$ & $0.0071(10)$ & $0.0033(10)$ & $0.0035(9)$ \\
C24 & $0.0153(14)$ & $0.0143(12)$ & $0.0171(12)$ & $0.0070(10)$ & $0.0076(10)$ & $0.0068(10)$ \\
C25 & $0.0157(14)$ & $0.0131(12)$ & $0.0174(13)$ & $0.0038(10)$ & $0.0065(11)$ & $0.0045(10)$ \\
C3 & $0.0132(13)$ & $0.0140(13)$ & $0.0146(12)$ & $0.0041(10)$ & $0.0046(10)$ & $0.0035(9)$ \\
O5 & $0.0151(10)$ & $0.0131(9)$ & $0.0279(11)$ & $0.0043(8)$ & $0.0079(8)$ & $0.0076(8)$ \\
O6 & $0.0143(10)$ & $0.0138(9)$ & $0.0319(11)$ & $0.0053(8)$ & $0.0113(9)$ & $0.0065(8)$ \\
N3 & $0.0156(12)$ & $0.0176(11)$ & $0.0150(11)$ & $0.0073(9)$ & $0.0063(9)$ & $0.0047(8)$ \\
C31 & $0.0109(13)$ & $0.0164(13)$ & $0.0197(13)$ & $0.0045(11)$ & $0.0033(11)$ & $0.0067(10)$ \\
C32 & $0.0121(13)$ & $0.0127(12)$ & $0.0220(13)$ & $0.0045(10)$ & $0.0043(11)$ & $0.0089(10)$ \\
C33 & $0.0137(13)$ & $0.0148(12)$ & $0.0108(11)$ & $0.0033(10)$ & $0.0038(10)$ & $0.0064(9)$ \\
C34 & $0.0111(13)$ & $0.0135(12)$ & $0.0159(12)$ & $0.0021(10)$ & $0.0058(10)$ & $0.0009(9)$ \\
C35 & $0.0165(14)$ & $0.0117(12)$ & $0.0201(13)$ & $0.0038(10)$ & $0.0079(11)$ & $0.0047(10)$ \\
& & & & &
\end{tabular}

Geometric parameters $\left(\AA,^{\circ}\right)$

\begin{tabular}{llll}
\hline $\mathrm{C} 1-\mathrm{O} 1$ & $1.215(3)$ & $\mathrm{C} 22-\mathrm{C} 23$ & $1.399(4)$ \\
$\mathrm{C} 1-\mathrm{O} 2$ & $1.320(3)$ & $\mathrm{C} 22-\mathrm{H} 22$ & 0.9300 \\
$\mathrm{C} 1-\mathrm{C} 2$ & $1.559(4)$ & $\mathrm{C} 23-\mathrm{C} 24$ & $1.398(4)$ \\
$\mathrm{O} 2-\mathrm{H} 1$ & 0.8200 & $\mathrm{C} 24-\mathrm{C} 25$ & $1.393(4)$ \\
$\mathrm{C} 2-\mathrm{O} 3$ & $1.226(3)$ & $\mathrm{C} 24-\mathrm{H} 24$ & 0.9300 \\
$\mathrm{C} 2-\mathrm{O} 4$ & $1.292(3)$ & $\mathrm{C} 25-\mathrm{H} 25$ & 0.9300 \\
$\mathrm{O} 4-\mathrm{H} 2$ & 0.8200 & $\mathrm{C} 3-\mathrm{O} 5$ & $1.220(3)$ \\
$\mathrm{N} 1-\mathrm{C} 11$ & $1.348(3)$ & $\mathrm{C} 3-\mathrm{O} 6$ & $1.299(3)$ \\
$\mathrm{N} 1-\mathrm{C} 15$ & $1.349(3)$ & $\mathrm{C} 3-\mathrm{C} 3$ & $1.563(5)$ \\
$\mathrm{C} 11-\mathrm{C} 12$ & $1.392(4)$ & $\mathrm{O} 6-\mathrm{H} 3$ & 0.8200 \\
$\mathrm{C} 11-\mathrm{H} 11$ & 0.9300 & $\mathrm{~N} 3-\mathrm{C} 35$ & $1.334(4)$ \\
$\mathrm{C} 12-\mathrm{C} 13$ & $1.408(4)$ & $\mathrm{N} 3-\mathrm{C} 31$ & $1.344(3)$
\end{tabular}




\begin{tabular}{|c|c|c|c|}
\hline $\mathrm{C} 12-\mathrm{H} 12$ & 0.9300 & $\mathrm{C} 31-\mathrm{C} 32$ & $1.388(4)$ \\
\hline $\mathrm{C} 13-\mathrm{C} 14$ & $1.404(3)$ & $\mathrm{C} 31-\mathrm{H} 31$ & 0.9300 \\
\hline $\mathrm{C} 13-\mathrm{C} 23$ & $1.497(4)$ & $\mathrm{C} 32-\mathrm{C} 33$ & $1.406(4)$ \\
\hline $\mathrm{C} 14-\mathrm{C} 15$ & $1.385(4)$ & $\mathrm{C} 32-\mathrm{H} 32$ & 0.9300 \\
\hline $\mathrm{C} 14-\mathrm{H} 14$ & 0.9300 & $\mathrm{C} 33-\mathrm{C} 34$ & $1.404(4)$ \\
\hline $\mathrm{C} 15-\mathrm{H} 15$ & 0.9300 & $\mathrm{C} 33-\mathrm{C} 33^{\mathrm{ii}}$ & $1.492(5)$ \\
\hline $\mathrm{N} 2-\mathrm{C} 25$ & $1.337(4)$ & $\mathrm{C} 34-\mathrm{C} 35$ & $1.389(4)$ \\
\hline $\mathrm{N} 2-\mathrm{C} 21$ & $1.350(3)$ & C34-H34 & 0.9300 \\
\hline $\mathrm{C} 21-\mathrm{C} 22$ & $1.386(4)$ & C35-H35 & 0.9300 \\
\hline $\mathrm{C} 21-\mathrm{H} 21$ & 0.9300 & & \\
\hline $\mathrm{O} 1-\mathrm{C} 1-\mathrm{O} 2$ & $125.6(3)$ & $\mathrm{C} 23-\mathrm{C} 22-\mathrm{H} 22$ & 120.3 \\
\hline $\mathrm{O} 1-\mathrm{C} 1-\mathrm{C} 2$ & $122.3(3)$ & $\mathrm{C} 24-\mathrm{C} 23-\mathrm{C} 22$ & $118.3(2)$ \\
\hline $\mathrm{O} 2-\mathrm{C} 1-\mathrm{C} 2$ & $112.1(2)$ & $\mathrm{C} 24-\mathrm{C} 23-\mathrm{C} 13$ & $120.1(2)$ \\
\hline $\mathrm{C} 1-\mathrm{O} 2-\mathrm{H} 1$ & 109.5 & $\mathrm{C} 22-\mathrm{C} 23-\mathrm{C} 13$ & $121.7(2)$ \\
\hline $\mathrm{O} 3-\mathrm{C} 2-\mathrm{O} 4$ & $126.4(3)$ & $\mathrm{C} 25-\mathrm{C} 24-\mathrm{C} 23$ & $118.5(3)$ \\
\hline $\mathrm{O} 3-\mathrm{C} 2-\mathrm{C} 1$ & $120.7(3)$ & $\mathrm{C} 25-\mathrm{C} 24-\mathrm{H} 24$ & 120.8 \\
\hline $\mathrm{O} 4-\mathrm{C} 2-\mathrm{C} 1$ & $112.9(2)$ & $\mathrm{C} 23-\mathrm{C} 24-\mathrm{H} 24$ & 120.8 \\
\hline $\mathrm{C} 2-\mathrm{O} 4-\mathrm{H} 2$ & 109.5 & $\mathrm{~N} 2-\mathrm{C} 25-\mathrm{C} 24$ & $123.1(2)$ \\
\hline $\mathrm{C} 11-\mathrm{N} 1-\mathrm{C} 15$ & $119.5(2)$ & $\mathrm{N} 2-\mathrm{C} 25-\mathrm{H} 25$ & 118.4 \\
\hline $\mathrm{N} 1-\mathrm{C} 11-\mathrm{C} 12$ & $121.9(3)$ & $\mathrm{C} 24-\mathrm{C} 25-\mathrm{H} 25$ & 118.4 \\
\hline $\mathrm{N} 1-\mathrm{C} 11-\mathrm{H} 11$ & 119.0 & $\mathrm{O} 5-\mathrm{C} 3-\mathrm{O} 6$ & $126.3(2)$ \\
\hline $\mathrm{C} 12-\mathrm{C} 11-\mathrm{H} 11$ & 119.0 & $\mathrm{O} 5-\mathrm{C} 3-\mathrm{C} 3^{\mathrm{i}}$ & $121.0(3)$ \\
\hline $\mathrm{C} 11-\mathrm{C} 12-\mathrm{C} 13$ & $119.2(2)$ & $\mathrm{O} 6-\mathrm{C} 3-\mathrm{C} 3^{\mathrm{i}}$ & $112.7(3)$ \\
\hline $\mathrm{C} 11-\mathrm{C} 12-\mathrm{H} 12$ & 120.4 & $\mathrm{C} 3-\mathrm{O} 6-\mathrm{H} 3$ & 109.5 \\
\hline $\mathrm{C} 13-\mathrm{C} 12-\mathrm{H} 12$ & 120.4 & $\mathrm{C} 35-\mathrm{N} 3-\mathrm{C} 31$ & $119.1(2)$ \\
\hline $\mathrm{C} 14-\mathrm{C} 13-\mathrm{C} 12$ & $117.8(2)$ & $\mathrm{N} 3-\mathrm{C} 31-\mathrm{C} 32$ & $122.3(3)$ \\
\hline $\mathrm{C} 14-\mathrm{C} 13-\mathrm{C} 23$ & $120.4(2)$ & $\mathrm{N} 3-\mathrm{C} 31-\mathrm{H} 31$ & 118.9 \\
\hline $\mathrm{C} 12-\mathrm{C} 13-\mathrm{C} 23$ & $121.8(2)$ & $\mathrm{C} 32-\mathrm{C} 31-\mathrm{H} 31$ & 118.9 \\
\hline $\mathrm{C} 15-\mathrm{C} 14-\mathrm{C} 13$ & $119.7(3)$ & $\mathrm{C} 31-\mathrm{C} 32-\mathrm{C} 33$ & $119.4(2)$ \\
\hline $\mathrm{C} 15-\mathrm{C} 14-\mathrm{H} 14$ & 120.1 & $\mathrm{C} 31-\mathrm{C} 32-\mathrm{H} 32$ & 120.3 \\
\hline $\mathrm{C} 13-\mathrm{C} 14-\mathrm{H} 14$ & 120.1 & $\mathrm{C} 33-\mathrm{C} 32-\mathrm{H} 32$ & 120.3 \\
\hline $\mathrm{N} 1-\mathrm{C} 15-\mathrm{C} 14$ & $121.8(2)$ & $\mathrm{C} 34-\mathrm{C} 33-\mathrm{C} 32$ & $117.3(2)$ \\
\hline $\mathrm{N} 1-\mathrm{C} 15-\mathrm{H} 15$ & 119.1 & $\mathrm{C} 34-\mathrm{C} 33-\mathrm{C} 33^{\mathrm{ii}}$ & $121.2(3)$ \\
\hline $\mathrm{C} 14-\mathrm{C} 15-\mathrm{H} 15$ & 119.1 & $\mathrm{C} 32-\mathrm{C} 33-\mathrm{C} 33^{\mathrm{ii}}$ & $121.5(3)$ \\
\hline $\mathrm{C} 25-\mathrm{N} 2-\mathrm{C} 21$ & $118.6(2)$ & $\mathrm{C} 35-\mathrm{C} 34-\mathrm{C} 33$ & $119.5(3)$ \\
\hline $\mathrm{N} 2-\mathrm{C} 21-\mathrm{C} 22$ & $122.1(3)$ & $\mathrm{C} 35-\mathrm{C} 34-\mathrm{H} 34$ & 120.2 \\
\hline $\mathrm{N} 2-\mathrm{C} 21-\mathrm{H} 21$ & 119.0 & $\mathrm{C} 33-\mathrm{C} 34-\mathrm{H} 34$ & 120.2 \\
\hline $\mathrm{C} 22-\mathrm{C} 21-\mathrm{H} 21$ & 119.0 & $\mathrm{~N} 3-\mathrm{C} 35-\mathrm{C} 34$ & $122.4(2)$ \\
\hline $\mathrm{C} 21-\mathrm{C} 22-\mathrm{C} 23$ & $119.5(2)$ & $\mathrm{N} 3-\mathrm{C} 35-\mathrm{H} 35$ & 118.8 \\
\hline $\mathrm{C} 21-\mathrm{C} 22-\mathrm{H} 22$ & 120.3 & C $34-\mathrm{C} 35-\mathrm{H} 35$ & 118.8 \\
\hline
\end{tabular}

Symmetry codes: (i) $-x+2,-y+2,-z+1$; (ii) $-x+3,-y+1,-z+1$.

Hydrogen-bond geometry $\left(\AA,{ }^{\circ}\right)$

\begin{tabular}{lllll}
\hline$D-\mathrm{H} \cdots A$ & $D-\mathrm{H}$ & $\mathrm{H} \cdots A$ & $D \cdots A$ & $D-\mathrm{H} \cdots A$ \\
\hline $\mathrm{O} 2-\mathrm{H} 1 \cdots \mathrm{N} 2^{\mathrm{iii}}$ & 0.82 & 1.77 & $2.586(3)$ & 174
\end{tabular}




$\begin{array}{lllll}\mathrm{O} 4-\mathrm{H} 2 \cdots \mathrm{N} 1 & 0.82 & 1.74 & 2.553(3) & 174 \\ \mathrm{O} 6-\mathrm{H} 3 \cdots \mathrm{N} 3 & 0.82 & 1.74 & 2.560(3) & 174 \\ \mathrm{C} 11-\mathrm{H} 11 \cdots 3^{2} & 0.93 & 2.59 & 3.270(4) & 130 \\ \mathrm{C} 22-\mathrm{H} 22 \cdots 3^{\mathrm{v}} & 0.93 & 2.45 & 3.375(3) & 172 \\ \mathrm{C} 24-\mathrm{H} 24 \cdots 1^{\mathrm{vi}} & 0.93 & 2.40 & 3.326(4) & 171 \\ \mathrm{C} 25-\mathrm{H} 25 \cdots{ }^{\mathrm{vi}} & 0.93 & 2.48 & 3.216(3) & 136 \\ \mathrm{C} 32-\mathrm{H} 32 \cdots 5^{\mathrm{vii}} & 0.93 & 2.56 & 3.418(3) & 153 \\ \mathrm{C} 34-\mathrm{H} 34 \cdots 5^{\mathrm{v}} & 0.93 & 2.52 & 3.373(3) & 153 \\ \mathrm{C} 35-\mathrm{H} 35 \cdots \mathrm{O}^{\mathrm{vii}} & 0.93 & 2.57 & 3.207(3) & 126\end{array}$

Symmetry codes: (iii) $x-1, y+1, z$; (iv) $-x,-y+1,-z$; (v) $x+1, y, z$; (vi) $x, y-1, z$; (vii) $-x+2,-y+1,-z+1$; (viii) $-x+3,-y+2,-z+1$. 